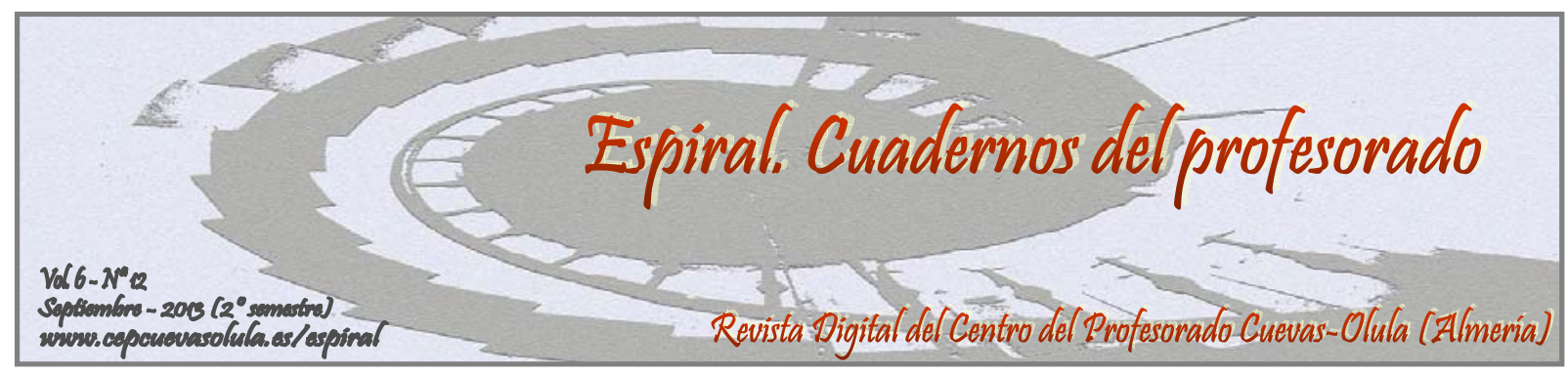

\title{
FORMACIÓN PERMANENTE DEL PROFESORADO Y PRÁCTICA DOCENTE INTERCULTURAL: CONTENIDOS ACTITUDINALES Y COMPLEMENTARIEDAD COMPETENCIAL
}

\section{SERVICE TEACHER TRAINING AND INTERCULTURAL TEACHING PRACTICE: ATTITUDINAL CONTENTS AND INTEGRATION OF COMPETENCES}

\author{
Ernesto López Gómez ${ }^{(1)}$ y Eufrasio Pérez Navío ${ }^{(2)}$
}

(1) Universidad Nacional de Educación a Distancia, España

(2) Universidad de Jaén, España

RESUMEN: La formación permanente del profesorado es un elemento clave en el contexto educativo actual. Por ello, el principal objetivo de este trabajo es aportar un nuevo modelo de formación del profesorado, teniendo en cuenta su bagaje intercultural. Para ello, se propone un modelo de formación permanente del profesorado basado en las necesidades de los centros educativos desde un proceso de investigación, innovación y evaluación teniendo en cuenta la competencia intercultural. Dicha competencia emerge con fuerza, haciéndose imprescindible la formación y el desarrollo del profesorado. A partir de una metodología de investigación narrativa, se ha revisado la literatura científica de los principales autores destacando, como conclusión general, que ser docente en el siglo XXI implica un compromiso unánime y constante del profesorado para crear un clima apropiado de aprendizaje en el aula teniendo en cuenta la propia práctica docente y la adquisición y desarrollo de la competencia intercultural.

Palabras clave: investigación, innovación, profesorado, interculturalidad, actitud.

ABSTRACT: The main objective of this work is to provide a new training model considering the cultural background of teachers. For this, the teacher training should be an essential element in the current educational context. In this paper we propose a model of teacher training based on the needs of schools from a process of research, innovation and evaluation taking into account intercultural competence. Such competence emerges strongly, becoming essential to training and teacher development. Based on a narrative research methodology, has been reviewed scientific literature emphasizing, as a general conclusion, that being a teacher in the XXI century implies a unanimous and constant commitment of teachers to create an appropriate climate for learning in the classroom taking into account the own practice and the acquisition and development of intercultural competence.

Key words: research, innovation, teaching, intercultural, attitude.

López Gómez, E., y Pérez Navío, E. (2013). Formación permanente del profesorado y práctica docente intercultural: contenidos actitudinales y complementariedad competencial. Espiral. Cuadernos del Profesorado, 6(12), 32-42. Disponible en: http://www.cepcuevasolula.es/espiral.

Fecha de recepción: 11/03/2013

Fecha de aceptación: 30/07/2013
Enviar correspondencia a: elopezgeducacion@gmail.com 


\section{1.- INTRODUCCIÓN}

La interculturalidad emerge en las instituciones educativas como una oportunidad ineludible para la educación actual y requiere asumir como principio orientador la práctica educativa intercultural del profesorado. La importancia de este trabajo radica en los procesos de innovación docente, en la investigación-acción que el profesorado puede realizar en su práctica docente, en las actividades de formación individuales y/o colectivas que organizan los Centros de Profesores (CEP), el intercambio de información con otros docentes, la retroalimentación que reciban del alumnado, etc.

Si se trata de desarrollar perfiles de profesorado para ajustar el trabajo docente a las necesidades escolares (OCDE, 2004; Mourshed, Chijioke y Barber, 2010) abordar la formación intercultural del profesorado resulta sumamente pertinente como realidad deficitaria de la educación actual.

Así, la formación del profesorado en la actualidad ha de encontrar pleno sentido desde las prácticas que más vivifican el desarrollo docente de la nueva escuela y de sus urgentes necesidades. De este modo, la interculturalidad expresada desde contenidos formativos se vincula estrechamente con la actual realidad social y escolar, y requiere de un tratamiento formativo amplio que no se reduzca únicamente a la formación inicial del profesorado, sino que se proponga como capacitación permanente.

En este artículo se justifica la relevancia de la formación permanente del profesorado para el ejercicio de la práctica docente intercultural. Nuestro guión argumentativo parte de la premisa de que son la educación, la apertura al diálogo y el encuentro los principales ejes para el acercamiento de las culturas y, la escuela es el mejor escenario para ello. Solo así, desde la implicación y el compromiso del profesorado y formadores se podrá construir una fecunda educación intercultural que supere los límites de las instituciones educativas y se sitúe en la amplitud del hecho y fenómeno educativo (Leiva, 2008).

La formación permanente del profesorado en la interculturalidad consiste en un desarrollo de la competencia intercultural del profesorado construido sobre y desde las actitudes más valiosas y necesarias en el docente, así como en estrecha complementariedad con las competencias comunicativa, tecnológica y especialmente colaborativa, para una auténtica práctica educativa e intervención didáctica interculturales.

Por todo ello, el objetivo principal de este trabajo es aportar un nuevo modelo de formación permanente del profesorado, teniendo en cuenta el bagaje intercultural adquirido a través de diversas fuentes (práctica docente, cursos de formación, innovación colaborativa entre centros, comunidades de aprendizaje, actitud personal-profesional del docente, etc.). Para ello, se realiza una exposición teórica y narrativa, fruto de la revisión de autores relevantes que han aportado un horizonte de sentido en torno a la formación del profesorado desde una perspectiva intercultural.

\section{2.- LA APUESTA POR LA FORMACIÓN Y EL DIÁLOGO INTERCULTURAL EN LA AM- PLITUD DEL HECHO EDUCATIVO}

La interculturalidad ha de potenciar la interrelación entre la amalgama cultural presente en la sociedad -y también en las instituciones formativas-, que favorezca un continuo y fecundo diálogo entre todas ellas pero que no renuncie a su vez a la identidad original de cada una. Este diálogo entre culturas implica tender puentes y vínculos que promuevan una interacción cultural fructífera que contribuya al enriquecimiento de las personas. Así lo recoge también la UNESCO (2001, art.3) cuando hace más de una década indicaba que "la diversidad cultural amplía las posibilidades de elección que se brindan a todos; es una de las fuentes del desarrollo (...) de acceso a una existencia intelectual, afectiva, moral y espiritual satisfactoria". En efecto, la diversidad se configura como una fuente de variados sentidos y su presencia en la escuela más que un problema se convierte en un auténtico reto educativo (Jordán, 2007; Medina, Rodríguez e Ibáñez, 2005).

La educación intercultural es un compromiso con la diversidad. Como tal, ha de proyectarse en las instituciones educativas como uno de sus grandes principios, orientado a la mejora de las acciones y relaciones entre las culturas que participan en la comunidad educativa (Bernabé, 2012). 
La auténtica educación intercultural se construye mediante el diálogo y el encuentro compartido entre todos los estudiantes, docentes y demás seres humanos que participen de esa comunidad formativa y se proyecta como un proceso de intercambio creador y recíproco donde los involucrados interiorizan los aspectos más representativos de las culturas en evolución y síntesis permanente, mediante la que se logra una forma nueva de avanzar y de sentirse ante la realidad social tan plural del momento (Medina y Domínguez, 1999; Domínguez, 2006). Es tarea de la pedagogía elaborar una propuesta educativa que vaya más allá de la simple búsqueda de la eficacia en la transmisión de los elementos culturales mayoritarios, que busque los valores y compartidos y que su cultivo permitan la cohesión social (Ibáñez-Martín, Fuentes y Barrio, 2012; Arnáiz y Escarbajal, 2012).

Sin duda que esa idea de compartir y dialogar, como construcción y proyección, es el núcleo esencial de la interculturalidad y de su aplicación y vigencia en el ámbito educativo (Escarbajal, 2011). Este diálogo y búsqueda de interacción y avance constantes son los elementos diferenciadores de lo que habitualmente se conoce como multiculturalismo, o coexistencia independiente de culturas en un mismo lugar, e interculturalismo (García, 2005).

Disciplinas concretas como Didáctica y Organización Escolar son claves para ofrecer alternativas educativas que puedan dar cauce a la realidad sucedida en los últimos años, en los que las aulas de los centros educativos han visto crecer de manera exponencial el número de niños procedentes de múltiples nacionalidades en un periodo de tiempo relativamente corto (Bartolomé, 2002; Soriano, 2008).

Los principios organizacionales y las estrategias didácticas de intervención y afrontamiento de esta nueva situación en la escuela requieren de una sólida reflexión pedagógica y de la formación del profesorado en ellas, si lo que se pretende es una escuela actual inclusiva (Arnáiz, 2012). En efecto, la cultura institucional implica a todos los miembros de la comunidad educativa en el conocimiento riguroso de las culturas, de sus valores y problemas más representativos para desde la práctica integrada de la educación interculturalidad realizar el trasvase formativo y sincero de verdaderas aportaciones que cada cultura pretende sumar a las otras y cuya síntesis contribuye al desarrollo intercultural (Medina, Domínguez y Medina, 2010). Resulta fundamental entonces trabajar la cultura organizativa de las instituciones desde un clima colaborativo, como puente efectivo para el avance y la construcción del soporte donde desarrollar una auténtica práctica de la educación bajo la mirada intercultural.

Pero la educación intercultural, en su amplio sentido, no puede circunscribirse a la institución escolar o educativa. Al menos, por dos cuestiones. En primer lugar, el contacto entre culturas no es un hecho que se limite en exclusividad al contexto escolar-formal y en segundo lugar, todos son conscientes de que la educación no se reduce únicamente a los años de escuela y educación formal. Consecuentemente, el marco y el espacio de desarrollo de la educación intercultural debe incorporar en su discurso los procesos no formales -adquisición de educación mediante estímulos directamente educativos, en actividades no conformadas por el sistema escolar- o informales -adquisición de educación mediante estímulos no directamente educativos- (Touriñán, 2008, p. 46). Se necesita entonces de la colaboración intensa y del trabajo conjunto de otros agentes del ámbito de la educación no formal e informal capaces de actuar con gran efectividad integrando acciones educativas desde diversas ópticas (García, 1994). De lo contrario, se antoja difícil crear climas interculturales en la escuela, más aún mientras el alumnado vive en la calle la experiencia cotidiana de una sociedad fragmentada donde no se aceptan los valores vivenciados en los procesos interculturales (Bartolomé, 2003).

De lo dicho hasta ahora se deduce la pertinencia de la educación intercultural desde el diálogo entre las diversas culturas como núcleo central de los procesos educativos situados más allá de las instituciones educativas. Así, la interculturalidad se vivencia y proyecta no solo como síntesis superadora de culturas, sino básicamente como un auténtico estilo de vida basado en la complementariedad y la búsqueda de un nuevo horizonte de colaboración y sinergias entre todas ellas, aunque en algunas ocasiones con tensiones y relaciones de difícil armonía y de limitaciones 
conflictivas a los modos de compartir un auténtico proyecto de vida y búsqueda de sentido y mejora (Medina, Domínguez y Medina, 2010, p. 19). Se dibuja entonces un reto intercultural: "pensar en el ser humano como capaz de combinar la cultura universalizada y la circundante a él realizando desplazamientos situacionales de una a otra sin problemas en forma de avances porque su yo, multifacético, está inevitablemente abierto incluso a influencias procedentes de fuera de su contorno" (Touriñán, 2010, p. 24).

En definitiva, la educación intercultural requiere de un intercambio y diálogo continuo, de ida y vuelta constante, entre las gentes que viven dicho contraste vital en complejidad donde se haga fértil la tarea del educador. Apostar, por tanto, por una formación profunda del profesorado en la interculturalidad desde la que proyectar y construir una práctica docente auténtica y comprometida desde las actitudes más valiosas que promocionan la educación intercultural.

Asumir una perspectiva intercultural para la educación permite añadir un valor extra a las formas convencionales de aprender, situando al currículo como gran oportunidad de cambio, permitiendo a la educación adaptarse a las nuevas situaciones, gestionar el cambio de nuestras sociedades diversas y plurales, incorporando en definitiva al horizonte escolar y no escolar la pertinencia de un capital intercultural (Santos, 2011).

Son los jóvenes quienes disfrutan de nuestras escuelas y en sus manos está el devenir social y cultural por lo que son ellos, las futuras generaciones, quienes más necesitan de modelos plenos e integrales aportados desde el compromiso intercultural de los educadores y profesorado, en el amplio marco social, para que puedan construir y desarrollar proyectos de vida auténticos en coherencia con una sociedad en la que habitan multitud de culturas.

\section{3.- LA INCIPIENTE PREOCUPACIÓN POR LA FORMACIÓN PERMANENTE: TENDEN- CIAS ACTUALES HACIA LA INTERCULTURALIDAD}

Una mirada a la historia muestra que en cuestiones de formación permanente del profesorado ha habido avances sustanciales pese a llevar "pocos años" siendo un tema de reflexión e indagación pedagógica. En efecto, la formación permanente del profesorado se ha dibujado como un ámbito de posterior discusión a la formación inicial, pero en la actualidad, ante los avances tan rápidos y los cambios que está experimentando las instituciones formativas, la velocidad de expansión del conocimiento y el valor de la formación para su alcance, es necesario otorgarle un desarrollo prioritario.

Así, no pocas veces la formación permanente aparece minusvalorada por aquellos que creen que la formación inicial es capaz de "inmunizar" al profesorado ante los retos formativos presentes y futuros. Desde luego que la formación inicial es muy importante para el docente, como lugar de inmersión en la enseñanza, de aprendizaje, desarrollo y construcción de la identidad profesional, pero ante los avances de la escuela y en el continuo propósito del profesorado por dar respuesta a los retos de hoy se requiere de un sólido sistema formativo que capacite al docente a lo largo de su trayectoria profesional. Más aún para la etapa de secundaria donde como señala Jordán con gran acierto "quizás apremie proporcionalmente más la formación permanente del profesorado, dada la escasa preparación inicial normalmente recibida” (Jordán, 2007, p. 66).

Sparks y Loucks (1990) señalan que la preocupación por la formación permanente del profesorado comienza en la década de 1970 debido, en primer lugar, a la actitud abierta y positiva de los docentes y también a la necesidad de éstos por formarse en cuestiones diferentes a las que habían sido formados y a las que por entonces se proponían desde los centros de formación del profesorado. Aquel modelo de formación permanente emergió así caracterizado por ser un proceso en el cual el profesorado seguía las actividades formativas que consideraban que podían facilitarles algún aprendizaje, en definitiva, era un modelo formativo individual formulado bajo la premisa: "fórmate como puedas, y dónde puedas” (Imbernón, 2007, p. 19).

Fue durante la década de 1980 cuando se logró, en España, alcanzar la plena escolarización de las jóvenes generaciones (Ruiz, 2000; Esteve, 2003) y este avance cuantitativo supuso, obviamente, que las aulas se llenaron de estudiantes. Esto reclamó al profesorado de una formación distinta a la que 
por entonces recibían, una capacitación llamada a dar cauce a las necesidades emergentes en aquel momento muy centradas en la atención a los estudiantes en su diversidad como eje de la calidad educativa. Así desde el tema que aquí nos ocupa, la formación permanente, se incorporaron elementos técnicos como la planificación y programación de la enseñanza desde prácticas conductistas donde se valoraba con gran importancia la correcta redacción de los objetivos didácticos (Real Decreto 2112/1984). Parece que el paradigma de la racionalidad técnica se impone dominantemente y dibuja un perfil de profesorado como alguien eminentemente técnico, desde su intervención mediada entre el conocimiento científico y la práctica real (Pérez, 1991, p. 79).

El nuevo estilo de pensar y de hacer en el aula supera la propia racionalidad técnica y se corresponde con la propuesta del profesor reflexivo (Zeichner, 1983; Schön, 1983, trad. esp. 1992). Así se concibe al docente desde una orientación indagadora que va más allá de la adquisición de unas teorías y destrezas prácticas, valorando como cuestión clave el cambio actitudinal en el docente (Rodríguez y Gutiérrez, 1991, p. 38).

Con todo, el cambio durante la década de los noventa se guió por la búsqueda del profesional indagador aunque siguió predominando la racionalidad técnica en forma de preparación instrumental y entrenamientos a los docentes como eje de su formación permanente. Existe por tanto, escasa transferencia entre lo que el profesorado aprende y el trabajo práctico-contextualizado de dichos aprendizajes. Algunos aspectos positivos de estos años fue la preocupación del ámbito universitario por la formación permanente del profesorado (Imbernón, 2007, p. 24) y el florecimiento de una conciencia del profesorado por formarse y tomar un papel activo en su capacitación permanente, a la par que se desarrollan modelos formativos y aparecen análisis metodológicos de la práctica educativa como son la investigación acción y el cambio curricular. En esa línea indica Stenhouse (1987) que el docente, además de indagar, ha de investigar cuestionando su práctica, comprometiéndose con su labor y reflexionando con los colegas sobre las mejores formas de hacer y orientar su tarea hacia la mejora.

En esencia, lo que conforma a un docente como profesional de la enseñanza, a la vez que contribuye de modo trascendental a su propia formación y desarrollo, "es su capacidad para actuar a partir del análisis de contexto, la reflexión sobre su propia práctica, la aplicación de sus teorías implícitas y explícitas y la investigación aplicada como instrumento crítico de sus actuaciones" (Domínguez y Blázquez, 1999, p. 162).

En un tiempo más cercano al actual las temáticas para la formación permanente son muy diversas. Algunos temas recurrentes se refieren a la renovación en modelos y métodos didácticos con incidencia tecnológica así como cuestiones de organización escolar, educación para la salud y ambiental, formación profesional, convivencia y resolución de conflictos e interculturalidad (Jiménez, 2007). Otra temática de formación permanente tiene que ver con el desarrollo de la competencia cognitiva del docente con una especial incidencia y énfasis en los espacios de problemas educativos, la atención a los distintos discentes y sus diversos ritmos de desarrollo, a cómo combinar la dimensión cognitiva y emocional en la actuación del profesorado, a la importancia de anticiparse a los cambios decisivos de los procesos educativos, al pensamiento y la actuación conjuntamente con otros y por último, la necesidad de crear ideas y poner en práctica nuevas hipótesis y alternativas en el diagnóstico y solución de problemas más allá de la mera aplicación de respuestas rutinarias y eficientes (Vázquez, 2007, p. 50).

Por otro lado, los docentes demandan formación permanente en aspectos relacionados con metodologías didácticas y las nuevas tareas encomendadas al profesorado, al margen de las tradicionales dirigidas a la impartición de conocimientos. Así, se puede reseñar los aspectos relativos a la innovación educativa, la orientación del alumno en los procesos de enseñanza aprendizaje y de los conocimientos sobre las tutorías y las diferentes actividades del profesional de la educación para ayudar al alumno en los distintos campos de la actividad académica y profesional (García, 1998; López, 2013).

En esta misma línea de intereses y necesidades formativas de los docentes, más concretamente para la formación permanente, Jordán (2006) nos muestra cómo, efectivamente, se percibe un creciente número de docentes que anhelan formarse y prepararse para una situación escolar 
crecientemente multicultural. De igual modo, la investigación de Garreta (2004) en las etapas de primaria y secundaria con una muestra de 740 docentes presenta una realidad preocupante: el 58\% de ellos confesó no tener ninguna formación en educación intercultural, el $40 \%$ sólo la poseía a un nivel básico -fruto de algún curso sobre el tema- y sólo el $2 \%$ restante parecía poseer una preparación más profunda, a partir de algún postgrado o modalidad formativa de categoría similar. Otro aval que fundamenta esta tesis es el estudio etnográfico llevado a cabo en Tenerife sobre formación en competencia intercultural en el que se pone de manifiesto que el $85 \%$ del profesorado participante en el estudio muestran interés por recibir una buena formación en la dimensión intercultural para ponerla realmente en práctica. El 80\% considera, además, que las actividades formativas deben ayudarles a construir sus propias ideas al respecto, a partir de la investigación de su propia práctica y alrededor del $70 \%$ creen que no basta el trabajo voluntario en solitario (López y García, 2006). En efecto, es sumamente importante el desarrollo profesional en colaboración y sintonía con el resto de profesionales del centro educativo más allá del voluntarismo e iniciativa de algunos docentes muy implicados. Se necesita fomentar una cultura de colaboración donde la formación permanente en la interculturalidad puede ayudar a conseguir la puesta en marcha de modelos formativos que surgen de las propias necesidades del centro y suponen beneficios para el conjunto de la comunidad educativa. En definitiva la formación en la competencia intercultural es un tema pertinente y ha de ser abordado desde la capacitación permanente.

\section{4.- MODALIDADES FORMATIVAS, CONTENIDOS ACTITUDINALES Y COMPLEMEN- TARIEDAD COMPETENCIAL EN LA FORMACIÓN PERMANENTE DEL PROFESORA- DO PARA UNA PRÁCTICA INTERCULTURAL}

La forma en la que capacitar al profesorado de forma continua tiene una gran incidencia sobre la comunidad educativa y muy especialmente en el centro educativo, como lugar de trabajo y desarrollo profesional del docente que se forma. Así se piensa que el modelo formativo de capacitación permanente ha de proyectarse a partir de la propia dinámica formativa-educativa del centro, para lograr la máxima implicación docente. En efecto, no tiene sentido esperar cambios significativos en la cultura y dinámica del centro si son solo unos pocos docentes los que sienten interés y necesidad por formarse y si además, dichos modelos formativos son en tiempo y en espacio fuera del centro educativo. Así se consigue igualmente que la formación permanente se adapte a las necesidades y demandas de los centros de forma concreta ya que es en ellos donde el profesorado que se forma ejercerá su labor.

Por todo ello nos parece pertinente un modelo paradigmático de investigación-innovacciónevaluación capaz de mejorar la práctica educativa a la vez que posibilita la autoformación del profesorado a través de una reflexión compartida constante por todos los implicados. Esta investigación y reflexión sobre las acciones llevadas a cabo permite una orientación de las prácticas innovadoras del centro desde la colaboración entre docentes (Stenhouse, 1987). Hoy tiene gran vigencia aquella idea formulada en tiempos de la "reforma educativa" que señalaba la pertinencia de la búsqueda del docente en la innovación un estilo nuevo de acción y reflexión. Una acción que ayude a saber hacer con eficiencia, eficacia y dominio artístico-reflexivo (Eisner, 1992) y una reflexión fecunda y sosegada para descifrar las claves y las demandas que la sociedad reclama a la tarea educativa (Medina y Domínguez, 1989).

El modelo formativo anterior asume la pluralidad de métodos posibles, así resulta tan valiosa una conferencia por parte de un experto como unas jornadas de trabajo así como un curso intensivo o un seminario de reflexión docente. En definitiva ha de pensarse la formación permanente desde un enfoque donde puedan tener cabida distintas modalidades formativas (Jordán, 2007) asumiendo la participación de otros profesionales, colectivos docentes, servicios de la comunidad, administración educativas, entre otras, que pueden aportar una gran incidencia e impacto en la comunidad educativa con implicaciones en el contexto socio-cultural más cercano entendiendo de este modo, como ya se subrayó anteriormente, a la interculturalidad como un fenómeno que se proyecta además de en las instituciones formativas también fuera de ellas donde es imprescindible de igual manera la formación y capacitación continua (Donoso, Cabrera, Aneas, de Santos y Curós, 2009). 
El modelo de formación permanente basado en las necesidades del centro pone a la interculturalidad como uno de sus posibles y grandes protagonistas. Uno de los objetivos de esta formación ha de ser prioritariamente el desarrollo de la competencia intercultural docente cuya complejidad conceptual crece con la necesidad que se tiene de utilizar el concepto de manera adecuada.

Como es bien sabido, las competencias superan el concepto de habilidades e integran saberes teóricos, prácticos y sobre todo vivenciales e implican al ser humano en su saber hacer, ser, actuar y sentir para responder a las demandas que conectan su formación con el desempeño de una profesión. La competencia intercultural del docente vendría a ser entonces una síntesis combinada de saberes interculturales, prácticas, actitudes y valores que constituyen un óptimo compromiso para comprender y actuar en plena colaboración con las culturas presentes en la escuela y la sociedad (Domínguez, 2006).

Haciendo una síntesis de algunos trabajos (Medina y Domínguez, 1999; García, 2003; Jordán, 2007; Medina, Domínguez y Medina, 2010) cabe indicar que resulta clave cultivar la interculturalidad como línea de respeto y apoyo continuo a las formas de saber, actuar y ser de los seres humanos fomentando las prácticas docentes más valiosas. Para ello se requiere un hondo compromiso de las escuelas, las comunidades, el profesorado, los estudiantes y las familias ante los nuevos modos de compartir la existencia lo que implica el diseño de programas de auténtico respeto a cada ser humano en su proyecto vital, comunitario y de plena transformación desde una continua reflexión docente sobre el clima intercultural que se construye desde el sistema metodológico empleado y que sitúa el discurso y el compromiso intercultural en el núcleo de los procesos educativos (Yuen, 2010).

Entre la formación y la práctica profesional se sitúa el compromiso ético y actitudinal que el docente necesita cultivar, desde actitudes valiosas que avancen en el empeño de lograr una práctica intercultural. Así se destaca por su representatividad (García, 2003, Jordán, 2007; Touriñán, 2010) la importancia de analizar, reconocer y reflexionar sobre las propias actitudes ante la diversidad adoptando un talante crítico respecto a las ideas y practicas consolidadas en los centros educativos, a veces de forma rutinaria, a modo de cultura implícita. Esto requiere sustituir una visión de la interculturalidad 'problematizadora' por otra abierta al reto, a la potencialidad enriquecedora que la diversidad lleva implícita consigo tomando conciencia de que la diversidad debe ser la norma del quehacer docente, superando el mito convencional de la inexistente 'homogeneidad' del alumnado.

Igualmente se hace necesario adoptar una actitud de constante formación, así como una construcción continua de una coherente teoría de la educación intercultural que guíe la práctica educativa diaria que permita replantearse la tarea docente como algo más educativo que instructivo en todos los niveles educativos.

Especial lugar ocupan los tutores -y su acción tutorial- para evitar la clara tendencia de derivar al alumnado minoritario hacia otro profesional de la orientación. El trabajo docente en perspectiva intercultural ha de darse en auténtica colaboración de todo el profesorado del mismo centro, así como con otros profesionales o agentes educativos externos implicados. En definitiva, es importante en el docente mostrar auténticas actitudes de tolerancia, escucha, valoración y reconocimiento hacia los estudiantes diferentes a la cultura mayoritaria. 
Además de cultivar las actitudes descritas y de otros contenidos formativos referidos a los valores interculturales que subyacen de la competencia intercultural docente se hace necesario asumir como objeto de formación permanente esencial y con especial relación con la competencia intercultural, por sus implicaciones en la tarea educativa, a la competencia tecnológica, comunicativa y colaborativa, especialmente. Así la competencia comunicativa se explicita de diversas formas pero sustancialmente en el discurso y estilo docente. Como indica Sarramona (1983) debería trabajarse a fondo, desde la formación permanente, tres características básicas del docente en cuanto que comunicador que se refieren al dominio de las asignaturas que profesa, al conocimiento del alumno y del medio sociocultural en el que vive.

Igualmente importante es desarrollar una cultura de colaboración en los centros desde el diálogo profundo que permita descubrir los valores de cada cultura, para lo cual puede ser útil, en la sociedad actual, servirse de las emergentes comunidades virtuales de aprendizaje y de los incipientes recursos tecnológicos comprendiendo que la educación intercultural es una responsabilidad compartida (Touriñán, 2010).

La armonización y complementariedad de las competencias intercultural, comunicativa, tecnológica y colaborativa permite avanzar en el proceso de enseñanzaaprendizaje en una sociedad plural por lo que ha de ser prioritaria la implicación del profesorado en su formación permanente para lograr así una práctica profesional intercultural.

\section{5.- CONCLUSIÓN}

Resulta difícil pensar hoy que no se necesita una actualización permanente de los conocimientos del profesorado así como una nueva formación sobre elementos que resultan valiosos para la labor docente. Más aún en una sociedad como la actual donde apremia la celeridad del avance científico, las nuevas demandas del mundo laboral, la obsesión por la eficiencia, la globalización, la presencia masiva de inmigrantes en las escuelas, desigualdades sociales, etc. Quizá hace algunos años, bastantes por cierto, la erudición de conocimientos y la experiencia acumulada en la práctica servían, en cierto modo, para afrontar las exigencias de la enseñanza.

Los contenidos de la formación permanente han de ser variados, desde las competencias más específicas e instrumentales hasta el desarrollo de actitudes valiosas para entender los procesos interactivos e interculturales vividos en el aula (Medina, 1999). En efecto, cada vez existe más diversidad en las aulas y la multiculturalidad, como modalidad de esa diversidad, es cada vez más significativa en nuestros centros educativos. Además, la dimensión puramente académica del profesorado y de su tarea resulta ya limitada. Estas consideraciones, entre otras, son aceptadas por la comunidad educativa y evidencia a su vez la necesidad de formación pedagógica que tiene el profesorado de todos los ciclos educativos, especialmente los que tradicionalmente han carecido de ella más notoriamente, como es el caso de los dedicados a la etapa de educación secundaria (Jordán, 2007; Pérez, 2009). Si queda suficientemente justificado que la educación intercultural es un propósito ineludible al que debe responder hoy en día la vida de todos los centros educativos es fundamental "indagar en la riqueza de la humanidad y en el necesario desarrollo de metodologías y acciones educativas inclusivas e interculturales” (Leiva, 2010, p. 55)

La formación docente en la interculturalidad requiere del análisis de los diversos contextos socioculturales y en las estrategias metodológicas que aborden la diversidad intercultural desde un profundo sentido de la tarea educativa en cada uno de los ámbitos de intervención socioeducativa, se resalta como grandes ámbitos de formación el saber ser y hacer desde un compromiso sentido con su tarea de educar seres humanos.

Una formación permanente que se caracterice por su emergencia y urgencia, sobre procesos de interacción continua entre teoría y práctica a través de la observación, investigación, innovación, reflexión y evaluación sobre la misma, llevada a cabo en el contexto institucional, donde los docentes desempeñan su labor (Imbernón, 2013). 
La idea de la formación permanente -la long life education docente- conecta con el aprendizaje a lo largo de la vida y con la necesidad del profesorado de estar continuamente formándose. La formación permanente del profesorado ha de favorecer la reflexión sobre su práctica potenciando en el docente un proceso de autoevaluación constante que le permita innovar y mejorar para adaptarse a la dinámica social y al entorno complejo que rodea al sistema educativo. La formación del profesorado, además, ha de cultivar en el docente el desarrollo de las actitudes más valiosas que les permitan comprender la interacción de aula y especialmente ante la realidad pluricultural en la que hoy se convive, actitudes de apertura a lo distinto y de respeto y valor hacia aquellas formas de ser y estar en el mundo diferentes.

Ser profesional de la educación y la cultura profesional docente han de sustantivarse de una metodología docente con un profundo compromiso actitudinal y con los valores más importantes de las personas que esperan formarse más allá del aprendizaje de unos conocimientos científicos (Leiva, 2011). Implica el manejo de las emociones, los vínculos afectivos entre el profesorado y anima a desarrollar una autoestima docente.

Hay que abandonar la concepción individualista de la profesión docente por otra donde exista una auténtica implicación, comunicación y colaboración del profesorado evitando así el aislamiento docente. La formación permanente que realmente proporciona a la profesión docente de su necesaria identidad es aquella que considera al docente como investigador de su propia enseñanza. Esta investigación requiere de formación previa y de innovación posterior que producirá una mejora y perfeccionamiento de los procesos formativos (Santos Guerra, 2010, p. 195). La consecuencia será clara: una formación permanente actual y adaptada a cada institución que permitirá avanzar en el desarrollo de las competencias docentes (Gairín, 2011).

Se ha de dibujar, en definitiva, una formación permanente que avance los retos, que supere problemas y por ello ha de incidir en aquellas situaciones cotidianas problemáticas de los centros educativos que ofrezcan una nueva perspectiva docente y formativa al profesorado.

\section{6.- REFERENCIAS}

Arnáiz, P. (2012). Escuelas eficaces e inclusivas: cómo favorecer su desarrollo. Educatio siglo XXI: Revista de la Facultad de Educación, 30(1), 25-44.

Arnáiz, P., y Escarbajal, A. (2012). Reflexiones sobre cultura, identidad y racismo desde una mirada pedagógica. Teoría de la Educación, 24(2), 83-106.

Bartolomé, M. (2002). Identidad y ciudadanía: un reto a la educación intercultural. Madrid: Narcea.

Bartolomé, M. (2003). Educación intercultural y ciudadanía. Aula Intercultural. Disponible en: http://www.aulaintercultural.org/IMG/pdf/bartolome-3.pdf

Bernabé, M. (2012). La comunicación intercultural a través de la música. Espiral. Cuadernos del Profesorado, 5(10), 87-97.

Domínguez, Ma . C. (2006). Investigación y formación del profesorado en una sociedad intercultural. Madrid: Universitas.

Domínguez, Mª. C., y Blázquez, F. (1999). Focos conceptuales para la formación inicial del profesorado de secundaria. Educación XX1, 2, 155-182.

Donoso, T., Cabrera, F., Aneas, A., de Santos, J. y Curós, P. (2009). Análisis de necesidades de formación intercultural en la Administración Pública. Revista de Investigación Educativa, 27(1), 149-167.

Eisner, E. W. (1992). La incomprendida función de las artes en el desarrollo humano. Revista Española de Pedagogía, 191, 15-34.

Escarbajal, A. (2011). Hacia la educación intercultural. Pedagogía Social: Revista Interuniversitaria, 18, 131149.

Esteve, J. M. (2003). La tercera revolución educativa. La educación en la sociedad del conocimiento. Barcelona: Paidós.

Gairín, J. (2011). Formación de profesores basada en competencias. Bordón. Revista de Pedagogía, 1 (63), 93108. 
García, J. L. (2005). Educación intercultural en Europa: un estudio comparado. En A. Medina, A. Rodríguez, Y A. Ibáñez, A. (coords.). (2005). Interculturalidad, formación del profesorado y educación (pp. 13-26). Madrid: Pearson.

García, J. L. (1998). La formación permanente del profesorado: motivaciones, realizaciones y necesidades. Educación XX1, 1, 129-158.

García, R. (2003). Formación del profesorado en pedagogía intercultural: contenidos actitudinales. Estudios Sobre Educación, 4, 47-65.

García, A. (1994). La educación intercultural en los ámbitos no formales. Documentación Social, 97, 147-160.

Garreta, J. (2004). El espejismo intercultural: la escuela de Cataluña ante la diversidad cultural. Revista de Educación, 333, 463-480.

Ibáñez-Martín, J. A., Fuentes, J. L., y Barrio, J. Mª (2012). Competencias sociales e inmigración desde una perspectiva intercultural. Educación XXI, 15(2), 41-72.

Imbernón, F. (2007). La formación permanente del profesorado. Nuevas ideas para formar en la innovación y el cambio. Barcelona: Graó (Ideas Clave).

Imbernón, F. (2013). Formación y desarrollo de la profesión, ¿de qué hablamos? Aula de Innovación Educativa, 218, 12-15.

Jiménez, B. (2007). La formación permanente que se realiza en los centros de apoyo al profesorado. Educación XX1, 10, 159-178.

Jordán, J. A. (2006). La escuela multicultural. Un reto para el profesorado. Barcelona: Paidós.

Jordán, J. A. (2007). Formación intercultural del profesorado de secundaria. Estudios Sobre Educación, 12, 5980.

Leiva, J. J. (2008). La escuela como espacio privilegiado para el desarrollo de la educación intercultural: el compromiso de los profesores. Campo Abierto, 27(1), 13-35.

Leiva, J. J. (2010). La educación intercultural entre el deseo y la realidad: reflexiones para la construcción de una cultura de la diversidad en la escuela inclusiva. Revista Docencia e Investigación, 20, 149-182.

Leiva, J. J. (2011). La educación intercultural en una encrucijada de caminos: reflexiones pedagógicas para la construcción de una escuela intercultural. Espiral. Cuadernos del Profesorado, 4(7), 43-56.

López, E. (2013). Aproximación a la percepción y satisfacción del profesor tutor de Secundaria Obligatoria respecto a su labor. Revista de Investigación en Educación, 11(1), 77-96.

López, P., y García, J. A. (2006). Sentimientos, sistema de creencias y comportamiento didáctico: un estudio etnográfico. Revista de Educación, 339, 493-516.

Medina, A., Rodríguez, A., e Ibáñez (coords.). (2005). Interculturalidad, formación del profesorado, y educación. Madrid: Pearson.

Medina, A., y Domínguez, Mª C. (1989). La formación del profesorado en una sociedad tecnológica. Madrid: Cincel.

Medina, A., Domínguez, Ma . C. (1999). Formación del profesorado: aprendizaje profesional en contextos interculturales. XX1 Revista Educación. Universidad de Huelva, 1, 69-97.

Medina, A., Domínguez, Mª C., y Medina, C. (2010). Tendiendo puentes hacia la interculturalidad: las nuevas escuelas y docentes. En F. Sadio (ed.). Tendiendo puentes hacia la interculturalidad (pp. 19-50). Granada: Ediciones K\&L.

Mourshed, M., Chijioke, C., y Barber, M. (2010). How the world's most improved school systems keep getting better. London: McKinsey \& Company Report.

OCDE (2004). Teachers matter: attracting, developing and retaining effective teachers. París: OCDE. Disponible en: http://www.oecd.org/dataoecd/38/36/34991371.pdf [Consulta: 8/12/2012].

Pérez, A. I. (1991). Investigación-acción y currículum. Revista Interuniversitaria de Formación del Profesorado, $10,69-84$.

Pérez, E. (2009). Procesos y Contextos Educativos. Jaén: Joxman Editores

Real Decreto 2112/1984, de 14 de noviembre, por el que se regula la creación y funcionamiento de los Centros de Profesores. Disponible en: http://www.boe.es/boe/dias/1984/11/24/pdfs/A33921-33922.pdf

[Consulta: 5/12/2012]. 
Rodríguez, A., y Gutiérrez, I. (1991). Necesidades de formación permanente del profesorado ante la Reforma de las enseñanzas no universitarias. Revista Interuniversitaria de Formación del Profesorado, 12, 33-42.

Ruíz, E. (2000). Un primer balance de la educación en España en el s. XX. Cuadernos de Historia Contemporánea, 22, 159-182.

Sarramona, J. (1983). La educación como sistema de comunicación (pp. 43-62). En J. L. Castillejo et al., Teoría de la educación I: El problema de la educación. Murcia: Límites.

Santos, M. A. (2010). Una pretensión problemática: educar para los valores y preparar para la vida. Revista de Educación, 351, 190-210.

Santos, M. A. (2011). Sostenibilidad y educación intercultural. El cambio de perspectiva. Bordón. Revista de Pedagogía, 63 (4), 123-135.

Schön, D. (1983). The Reflective Practitioner. How professionals think in action. London: Temple Smith. (trad. esp. 1992).

Sparks, D., y Loucks, S. (1990). Models of staff development (pp. 234-250). En W. R. Houston (ed.). Handbook of Research of Teacher Education. New York: MacMillan.

Soriano, E. (2008). Educar para la ciudadanía intercultural y democrática. Madrid: La Muralla.

Stenhouse, L. (1987). La investigación como base de la enseñanza. Madrid: Morata.

Touriñán J. M. (2008). El reto de la educación intercultural como ejercicio de la educación en valores (pp. 3552). En J. M. Touriñán (dir.) Educación en valores, educación intercultural y formación para la convivencia pacífica. La Coruña: Netbiblo.

Touriñán, J. M. (2010). Familia, escuela y sociedad civil. Agentes de educación intercultural. Revista de Investigación en Educación, 7, 7-36.

UNESCO (2001). Declaración Universal de la UNESCO sobre la diversidad cultural. Disponible en: http://portal.unesco.org/es/ev.phpURL_ID=13179\&URL_DO=DO_TOPIC\&URL_SECTION=201.html [Consulta: 8/12/2012].

Vázquez, G. (2007). La formación de la competencia cognitiva del profesor. Estudios Sobre Educación, 12, 4157.

Yuen, C. Y. (2010). Dimensions of diversity: challenges to secondary school teachers with implications for intercultural teacher education. Teaching and Teacher Education, 26, 732-741.

Zeichner, K. (1983). Alternative paradigm of teacher education. Journal of Teacher Education, 3, 3-9.

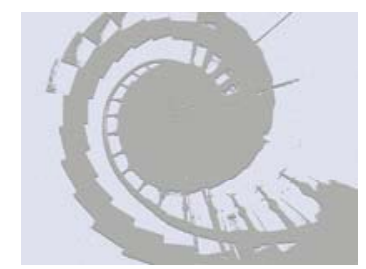

